\title{
Fractal-based models for the unsaturated soil hydraulic functions
}

\author{
M.A. Alfaro Soto ${ }^{\mathrm{a}, *}$, H.K. Chang ${ }^{\mathrm{a}}$, M.Th. van Genuchten ${ }^{\mathrm{b}}$ \\ a Departamento de Geologia Aplicada and Centro de Estudos Ambientais, UNESP - Universidade Estadual Paulista, Brazil \\ ${ }^{\mathrm{b}}$ Federal University of Rio de Janeiro, UFRJ, Rio de Janeiro, Brazil
}

A R T I C L E I N F O

Handling Editor: Morgan Cristine L.S.

Keywords:

Relative permeability

Fractal geometry

Water retention curve

Hydraulic conductivity

\begin{abstract}
A B S T R A C T
Reliable estimates of the unsaturated soil hydraulic properties are needed in many research and engineering projects. Fractal based approaches have become popular for describing the hydraulic functions. New models based on fractal geometry are presented to describe the wetting phase relative permeability (unsaturated hydraulic conductivity) of porous media. Analytical expressions were derived using the fractal capillary pressure (water retention) model proposed by Alfaro Soto and Vilar (2006) and the statistical pore size distribution models of Mualem (1976a) and Burdine (1953) for the unsaturated hydraulic conductivity. The newly derived models provide predictions of the relative permeability to water, as well as of the fractal dimension for modeling the pore structure of a soil. Results are compared with previous formulations by Brooks and Corey (1964), van Genuchten (1980) and Kosugi (1996), as well as with experimental water retention and hydraulic conductivity data of four soils having widely different soil textures. The proposed models agreed closely with observed data. The analytical models may be attractive alternative for estimating the unsaturated hydraulic conductivity when no laboratory or field measurements are available.
\end{abstract}

\section{Introduction}

Estimates of the unsaturated soil hydraulic conductivity are needed in various branches of the earth sciences, including civil and petroleum engineering, hydrogeology, soil science, vadose zone hydrology, and environmental and geotechnical engineering. Although the unsaturated hydraulic conductivity can be measured using a range of laboratory and field tests (e.g., Dane and Topp, 2002), the required experiments are generally too expensive, complex, and time-consuming for routine applications, especially when the conductivity is very low. Moreover, results can vary considerably if such factors as the wetting and drainage history of the porous medium or specific characteristics of the fluid (water, air, non-aqueous phase liquid) or medium are not considered in the tests. These various limitations have encouraged the use of indirect methods involving both empirical equations as well as more processbased formulations to determine the unsaturated hydraulic conductivity, $K$, as a function of the pressure head, $h$, or the water content, $\boldsymbol{\theta}$ (Brooks and Corey, 1964; Durner, 1994; Vereecken et al., 2010).

Statistical pore-size distribution models have made it possible to estimate the $K(\theta)$ or $K(h)$ functions based on the characteristics of the soil water retention (or capillary pressure - saturation) curve, $\theta(h)$. A range of models can be used for this, as exemplified by theoretical formulations by Purcell (1949), Childs and Collis-George (1950), Burdine (1953), Millington and Quirk (1961) and Mualem (1976a), among others. Using these theories, various analytical models have been obtained for the hydraulic conductivity functions, such as the widely used models of Brooks and Corey (1964) and van Genuchten (1980).

Fractal geometry has been used also for describing the hydraulic properties of unsaturated porous media. Tyler and Wheatcraft (1990) determined the hydraulic conductivity functions using a fractal model for the water retention curve, along with the statistical pore-size distribution models of Burdine (1953) and Mualem (1976a). Rieu and Sposito (1991) combined fractal descriptions of the water retention curve and the particle size distribution to obtain the fractal dimension necessary for calculating the hydraulic conductivity. Other contributions were fractal formulations of the porous medium where used in combination with predictive pore-size distribution approaches for the permeability are given by Fuentes et al. (1996), Xu (2004) and Li (2010), among others.

In this paper we propose an alternative fractal-based description of the unsaturated soil hydraulic functions using the conductivity models of Burdine (1953) and Mualem (1976a). Below we first briefly summarize various formulations for the hydraulic properties based on both traditional (mostly empirical) and fractal-geometric descriptions of the hydraulic properties. These descriptions are then compared with the new fractal-based formulations.

\footnotetext{
* Corresponding author.

E-mail addresses: alfaro@rc.unesp.br (M.A. Alfaro Soto), chang@rc.unesp.br (H.K. Chang).
} 


\section{Existing models for the soil hydraulic properties}

\subsection{Traditional analytical formulations}

A large number of empirical equations and statistical pore size distribution models have been used for the hydraulic properties of unsaturated media, including functions proposed by Brooks and Corey (1964), Campbell (1974), van Genuchten (1980), Fredlund et al. (1994), Kosugi (1996), and Assouline and Tartakovsky (2001). A detailed overview of various models is provided by Leij et al. (1997). Here we briefly summarize only those formulations that relate immediately to the work discussed in this paper.

Most of the predictive equations for the hydraulic conductivity are based on the statistical pore-size distribution models of Burdine (1953) or Mualem (1976a), which are given by

$K_{r}\left(S_{e}\right)=S_{e}^{2} \int_{0}^{S_{e}} \frac{d \tau}{h^{2}(\tau)} / \int_{0}^{1} \frac{d \tau}{h^{2}(\tau)}$

and

$K_{r}\left(S_{e}\right)=S_{e}^{0.5}\left[\int_{0}^{S_{e}} \frac{d \tau}{h(\tau)} / \int_{0}^{1} \frac{d \tau}{h(\tau)}\right]^{2}$

respectively, where $K_{r}=K(h) / K_{s}$ is the relatively hydraulic conductivity, $K_{s}$ is the saturated hydraulic conductivity, $h$ is the pressure head (for notational convenience assumed here to be positive for unsaturated conditions), and $S_{e}$ is effective saturation of the wetting phase:

$S_{e}(h)=\left(\theta(h)-\theta_{r}\right) /\left(\theta_{s}-\theta_{r}\right) \equiv\left(S(h)-S_{r}\right) /\left(1-S_{r}\right)$

in which $S\left(=\theta / \theta_{s}\right)$ is relative saturation, $S_{r}\left(=\theta_{r} / \theta_{s}\right)$ is residual saturation, and $\theta, \theta_{s}$ and $\theta_{r}$ are the volumetric, saturated and residual water contents, respectively.

Most early models for the hydraulic properties used a simple power expression for the water retention curve, exemplified by the classical model of Brooks and Corey (1964), which can be combined with Burdine's model (Eq. (1)) to yield the following set of hydraulic functions

$S_{e}(h)=\left(h_{a} / h\right)^{\lambda}$

$K_{r}\left(S_{e}\right)=S_{e}^{3+2 / \lambda}$

in which $h_{a}$ (generally referred to as the air entry value or bubbling pressure) and $\lambda$ (also known to as the pore size distribution index) are essentially empirical parameters.

Eq. (4) can be combined also with Mualem's model (Eq. (2)) to give

$K_{r}\left(S_{e}\right)=S_{e}^{5 / 2+2 / \lambda}$

Limitations often associated with Eqs. (4), (5) and (6) are the sharp breaks in the $S_{e}(h)$ and $K_{r}(h)$ curves at the air entry value, the absence of an inflection point, and possibly poor fits with retention data in the dry range. Some of these limitations motivated van Genuchten (1980) to introduce a smooth (continuous differentiable) water retention curve of the form

$S_{e}(h)=\left[1+(\alpha h)^{n}\right]^{-m}$

in which $\alpha, m$ and $n$, similarly as $h_{a}$ and $\lambda$ in Eq. (4), are also empirical parameters.

Eq. (7) with only minimal restrictions on permissible values of $m$ and $n$ (i.e., $n>1$, and $m>0$ ) can be combined directly with the Burdine and Mualem predictive equations to obtain very general but relatively complicated mathematical expressions for the hydraulic conductivity functions (van Genuchten and Nielsen, 1985; Dourado Neto et al., 2011). With the restriction $m=2-1 / n$ on permissible values of $m$ and $n$, the Burdine-based equations for the water retention and hydraulic conductivity functions are obtained by substituting Eq. (7) into Eq. (1) and integrating to yield (van Genuchten, 1980)
$K_{r}\left(S_{e}\right)=S_{e}^{2}\left[1-\left(1-S_{e}^{1 / m}\right)^{m}\right](m=1-2 / n)$

The Mualem-based equation for $K_{r}\left(S_{e}\right)$ is obtained similarly by substituting Eq. (7) into Eq. (2) and assuming $m=1-1 / n$ on permissible $m$ and $n$ values, to give

$K_{r}\left(S_{e}\right)=S_{e}^{0.5}\left[1-\left(1-S_{e}^{1 / m}\right)^{m}\right]^{2} \quad(m=1-1 / n)$

Another set of equations was derived by Kosugi (1996) who proposed a water retention curve assuming a log-normal pore radius distribution, to obtain

$S_{e}(h)=\frac{1}{2} \operatorname{erfc}\left\{\ln \left(h / h_{0}\right) / \sigma \sqrt{2}\right\}$

in which $h_{o}$ and $\sigma$ are parameters characterizing the log-normal poresize distribution, and erfc is the complementary error function. Kosugi (1996) combined Eq. (10) with both Eqs. (1) and (2) to obtain

$K_{r}(h)=\frac{1}{2} S_{e}^{2} \operatorname{erfc}\left[\ln \left(h / h_{0}\right) / \sigma \sqrt{2}+\sigma \sqrt{2}\right]$

for the Burdine-based conductivity $K_{r}(h)$ function, and

$K_{r}(h)=\frac{1}{2} S_{e}^{0.5}\left\{\operatorname{erfc}\left[\ln \left(h / h_{0}\right) / \sigma \sqrt{2}+\sigma / \sqrt{2}\right]\right\}^{2}$

for the Mualem-based $K_{r}(h)$ expression.

\subsection{Fractal formulations for the hydraulic functions}

Tyler and Wheatcraft (1990) were among the first to determine the permeability of a porous medium to water using fractal mathematics. For this they combined a fractal model for the water retention curve with the statistical models of Burdine (1953) and Mualem (1976a). Many others have followed since, including Toledo et al. (1990), Rieu and Sposito (1991), Giménez et al. (1997), and Xu (2004), and various references therein. Most of the fractal-based functions are relatively simple power functions of the pressure head, which, if combined with Burdine's conductivity model (Eq. (1)), will yield equations for threedimensional media of the form

$S_{e}(h)=\left(h_{a} / h\right)^{3-D}$

$K_{r}\left(S_{e}\right)=S_{e}^{\frac{11-3 D}{3-D}}$

in which $D$ is the fractal dimension. Eqs. (13) and (14) are identical to the Brooks and Corey equations given by Eqs. (4) and (5) if the poresize distribution exponent $\lambda$ is redefined in terms of the fractal dimension as

$\lambda=3-D$

which has become a key parameters in many fractal-based soil and hydraulic property studies.

Eq. (13) can also by combined with Mualem's conductivity model (Eq. (2)), in which case the fractal based conductivity function becomes

$K_{r}\left(S_{e}\right)=S_{e}^{\frac{19-3 D}{6-2 D}}$

Eqs. (13), (14) and (16), with various assumptions, modifications and extensions, have been derived or used in many studies (Tyler and Wheatcraft, 1990; Toledo et al., 1990; Fuentes et al., 1996; Giménez et al., 1997; Ghanbarian-Alavijeh et al., 2010, Xu and Dong, 2004; Ghanbarian-Alavijeh and Hunt, 2012b, among others), with some producing different power functions, especially of Eqs. (14) and (16), while others interpreted $D$ alternatively as the fractal dimension of the pore radius, pore surface or particle size distribution. Also, some studies assumed applicability of the equations over the entire moisture domain (thus assuming that $\theta_{r}=0$ ), while others applied the equations over only a limited part (e.g., between the air entry value and the pressure head at the permanent wilting point $(15,000 \mathrm{~cm}$ or some other value)).

Similarly as the traditional Brooks and Corey $S_{e}(h)$ curve, Eq. (13) has limitations in terms of the sharp break of the curve at the air entry 
value, $h_{a}$, and the function not exhibiting a inflection point often observed in field data. For this reason several have derived alternative fractal-based expressions for the hydraulic properties (e.g., Cihan et al., 2009; Li, 2010). One is by Li (2010) who derived relative permeabilities using fractal modeling of the medium in combination with the predictive conductivity models of Purcell (1949) and Burdine (1953). The water retention curve used in his study was of the form (Li, 2010)

$h=h_{\max }\left(1-b S_{e}\right)^{-1 / \lambda}$

where

$b=1-\left(h_{a} / h_{\max }\right)^{-\lambda}$

in which, as before, $\lambda=3-D$, and where $h_{a}$ and $h_{\max }$ are the lower and upper cutoff values for Eq. (17), taken by Li (2010) as the air entry value and the pressure head at the residual water content, respectively. Eq. (17) was previously used also by Bird (1998), as well as by Perfect (1999) who assumed the residual water content to be zero. The inverse expression of Eq. (18) is given by (Bird, 1998; Perfect, 1999; Ghanbarian-Alavijeh and Hunt, 2012a):

$S_{e}=\left(h_{\max }^{-\lambda}-h^{-\lambda}\right) /\left(h_{\max }^{-\lambda}-h_{a}^{-\lambda}\right) \quad h_{a} \leq h \leq h_{\max }$

Using Eq. (17), Li (2010) derived a model for the relatively hydraulic conductivity of the wetting phases using the predictive conductivity model of Purcell (1949), leading to

$K_{r}=\left(1-\left(1-b S_{e}\right)^{\frac{2+\lambda}{\lambda}}\right) /\left(1-\beta^{\frac{2+\lambda}{\lambda}}\right)$

where $b=1-\beta$, and $\beta=\left(h_{a} / h_{\max }\right)^{-\lambda}$. One may verify that $K_{r}\left(S_{e}=0\right)=0$, and $K_{r}\left(S_{e}=1\right)=1$ in the above equations. Li (2010) showed for two media (Berea sand and a rock sample) that Purcell's model worked very well for the wetting phase.

These various results suggest that fractal geometry can be used to describe the water retention curve, as well as of the hydraulic conductivity to water. These issues are further explored in the next section by using fractal theory to describe the water retention and hydraulic conductivity functions.

\section{Alternative fractal model for the hydraulic functions}

In this section, the fractal model for the water retention curve developed by Alfaro Soto and Vilar (2006) is used to derive formulations for the unsaturated hydraulic conductivity function using the approaches of Mualem (1976a) and Burdine (1953).

\subsection{Fractal model for the water retention curve}

Alfaro Soto and Vilar (2006) expanded upon previous work by considering the pore-size distribution instead of the particle size distribution, and introducing an equivalent water retention curve which does not assume a gradual and continuous reduction in pore size. Their water retention model is of the form

$\theta(h)=\left\{\begin{array}{cc}\theta_{r}\left[1+\left(h / h_{o}\right)^{-\lambda}\right] & h>h_{a} \\ 1 & 0 \leq h \leq h_{a}\end{array}\right.$

where $\lambda=3-D$ as before, $\theta_{r}$ is the residual water content, $h_{o}$ is an semi-empirical parameter, and $h_{a}$ is the usual air entry value characterizing a break in the retention function near saturation typical of the Brooks and Corey (1964) formulation. This parameter is a function of $h_{o}$ and $\lambda$ as follows:

$h_{a}=h_{o}\left(S_{r} /\left(1-S_{r}\right)\right)^{1 / \lambda}$

In terms of effective $\left(S_{e}\right)$ and relative $(S)$ saturations, Eq. (21) can be written also in the forms

$S_{e}(h)=\left\{\begin{array}{cc}\left(S_{r} /\left(1-S_{r}\right)\right)\left(h / h_{o}\right)^{-\lambda} & h>h_{a} \\ 1 & 0 \leq h \leq h_{a}\end{array}\right.$
$S(h)=\left\{\begin{array}{cc}S_{r}\left[1+\left(h / h_{o}\right)^{-\lambda}\right] & h>h_{a} \\ 1 & 0 \leq h \leq h_{a}\end{array}\right.$

where $S(h)=\theta / \theta_{s}$ and $S_{r}=\theta_{r} / \theta_{s}$.

Alfaro Soto and Vilar (2006) derived Eq. (21), similarly as Tyler and Wheatcraft (1990), by using a Menger sponge algorithm for the fractal pore size distribution without any assumption about the geometry of mass and the solid-pore interface. The equation incorporates two portions of the water retention curve, one associated with initial desaturation of macropores and represented by the region where the fractal characteristics are evident (with the fractal dimension being within in the range $0 \leq D \leq 3$ ), while a second region assumes that the lower limit of the water content when $h \rightarrow \infty$ is equal to the residual water content, $\theta_{r}$.

We consider Eq. (21) to be a semi-empirical equation since the model is made to partially satisfy a fractal condition in that the fractal portion is invariant upon dilation and has fractal scaling as suggested by Tyler and Wheatcraft (1990). In other words, the system remains identical under different magnifications (Adler, 1992) and has a hierarchical structure with larger units containing successively smaller and smaller units (Giménez et al., 1997).

Fig. 1 shows $S(h)$ plots based on Eq. (21) using hypothetical values of 0.23 for $S_{r}$ and $0.5 \mathrm{~m}$ for $h_{o}$, with $D$ varying from 1.0 to 2.9. The plots reflect the fact that for given values of $h_{o}$ (in this case $0.5 \mathrm{~m}$ ) and $S_{r}$, the air entry value $h_{a}$ decreases as the fractal dimension increases. Please note also that all curves go through the point $\left(h_{o}, 2 S_{r}\right)=(0.5,0.46)$, which follows immediately from Eq. (21). In all cases, relative saturation decreases between pressure heads of $h_{o}$ and $h_{a}$, but this would occur over a wider pressure head range if the residual water content is allowed to decrease simultaneously when $D$ increases. The curves resemble to some extent typical Brooks and Corey retention curves, except that they do not exhibit straight lines on complete log-log plots of $S_{e}(h)$, but only in the dry pressure head range.

Fig. 2 shows similar plots for different values of residual saturation $\left(S_{r}\right)$, varying from 0.1 to 0.5 , while $h_{o}$ and $D$ are maintained at $0.50 \mathrm{~m}$ and 1.0, respectively. The plots show not only that, as expected, relative saturation at a given pressure head increases with an increase in $S_{r}$, but also with an increase in the air entry value, $h_{a}$, as implied by Eq. (22). The simultaneous increase in $S_{r}$ and $h_{a}$ is typical of the water retention curves of relatively fine-textured soils (e.g., Carsel and Parrish, 1988; Schaap et al., 2001).

The flexibility of Eq. (21) in describing water retention data of various soils is demonstrated in Fig. 3. The soils studied were Lamberg clay, Caribou silty loam, Rubicon sandy loam, and Gravelly sand G.E.9, with data sets obtained from the catalogue of Mualem (1976b). Results obtained with Eq. (21) are compared in Fig. 3 with those using the models of Brooks and Corey (1964), van Genuchten (1980) and Kosugi

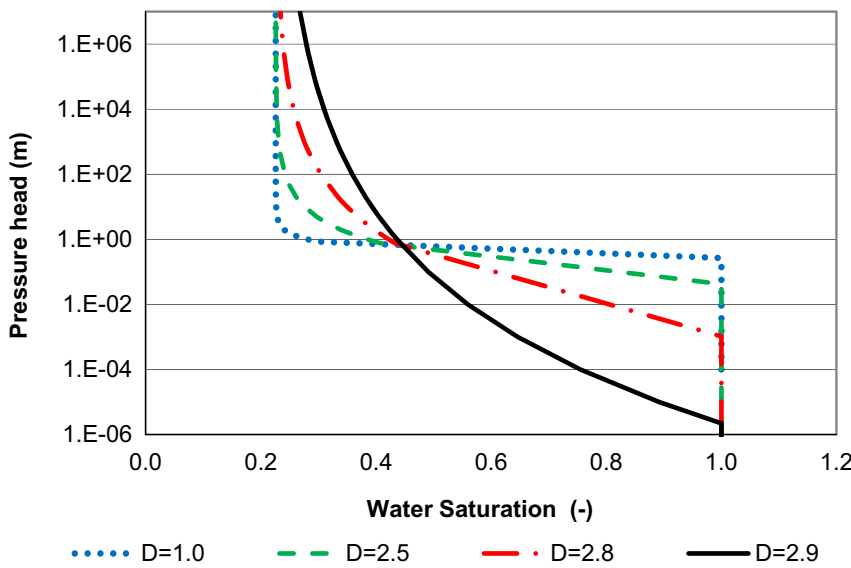

Fig. 1. Capillary pressure head - relative saturation curves calculated with Eq. (22) assuming $S_{r}=0.23$ and $h_{o}=0.50 \mathrm{~m}$, with $D$ varying from 1 to 2.9 . 


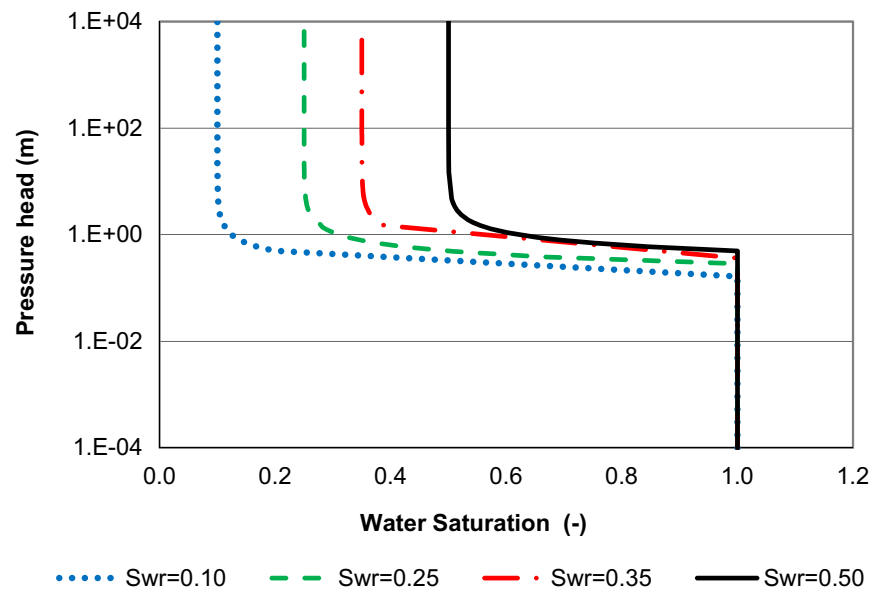

Fig. 2. Water pressure head - saturation curves calculated with Eq. (22) assuming $h_{o}=0.5 \mathrm{~m}$ and $D=1.0$, with $S_{r}$ varying between 0.10 and 0.50 .

(1996), while Table 1 presents the fitted parameters obtained using nonlinear regression. The fractal dimension varied between 0.70 and 2.61. No apparent relationship existed between texture and the value of $D$. However, all $D$ values were less than the theoretical upper limit 3.0 (Hunt, 2004).

The plots in Fig. 3 and the $R^{2}$ values in Table 1 indicate good agreement of Eq. (21) with the data. They show that results with Eq. (21) are very similar to those based on the models of Brooks and Corey

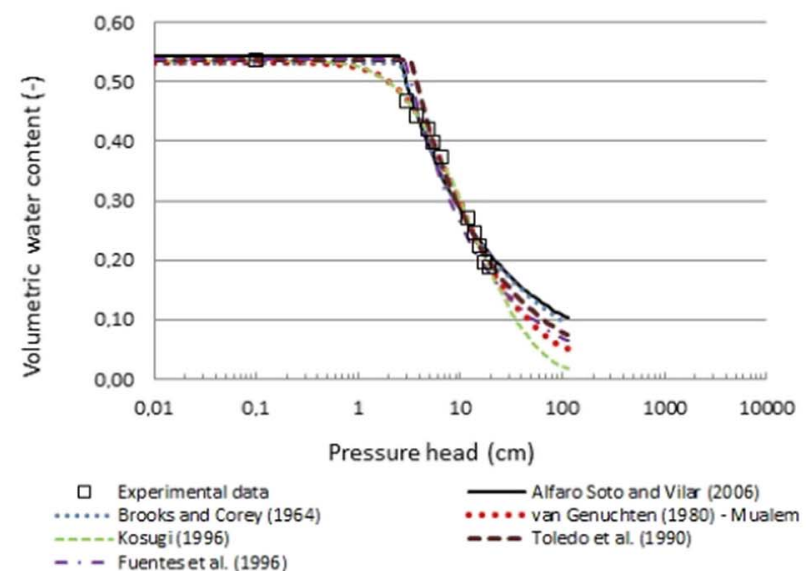

a

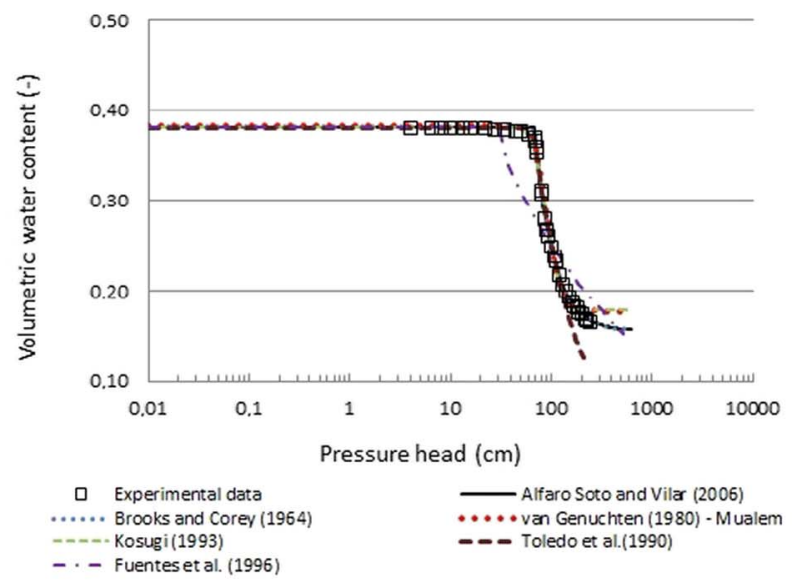

C
(1964), van Genuchten (1980) and Kosugi (1996). However, the plot for Lamberg clay in Fig. 3a revealed lower values for $R^{2}$, mostly because of less that optimal fits of the very wet and dry sides of the curve. The figure also shows that extrapolation of the fitted van GenuchtenMualem and Kosugi retention curves to the dry end did lead to lower residual water contents than the Brooks-Corey and our curves.

\subsection{Fractal models for the hydraulic conductivity}

We next used Eq. (21) or (23a) to derive predictive equations for the relative permeability $\left(K_{r}\right)$ using the models of Burdine (1953) and Mualem (1976a) as given by Eqs. (1) and (2), respectively. Solving Eq. (23a) for $h\left(S_{e}\right)$ gives

$h=h_{o}\left[S_{e} / S_{r}\right] \frac{1}{D-3}$

Substituting Eq. (24) into Eq. (1) and carrying out the integrations yields the relative permeability model based upon Burdine's approach:

$K_{r}\left(S_{e}\right)=S_{e}^{\frac{11-3 D}{3-D}}$

Similarly, the relative permeability of the wetting phase can be calculated using the model of Mualem (1976a) by substituting Eq. (24) into Eq. (2) and integrating to yield

$K_{r}\left(S_{e}\right)=S^{\frac{19-5 D}{6-2 D}}$

The remarkable result here is that Eqs. (25) and (26) exactly duplicate the Brook-Corey fractal based $K_{r}\left(S_{e}\right)$ expressions given by Eqs. (14) and (16). This even though different soil water retention models

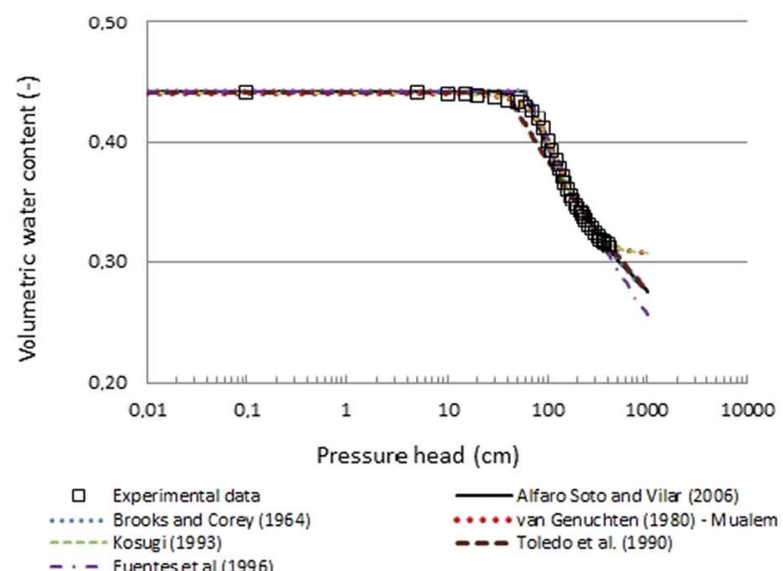

b

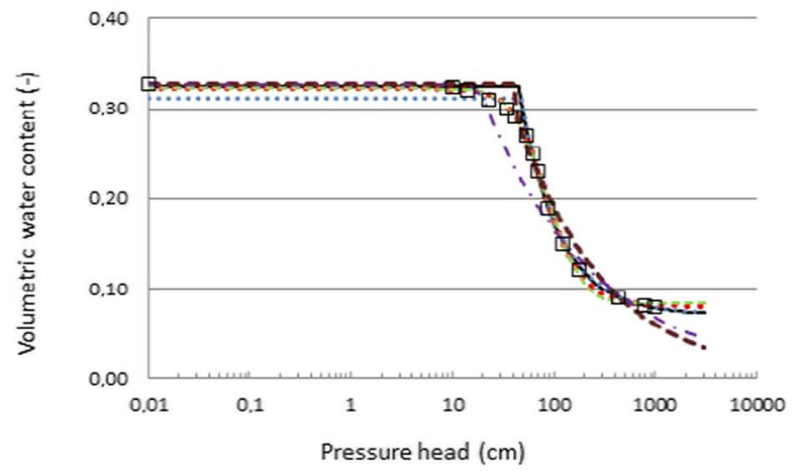

ㅁ. Experimental data

Alfaro Soto and Vila (2006) ...... Brooks and Corey (1964) …van Genuchten (1980) - Mualem - - Fuentes et al. (1996)

d

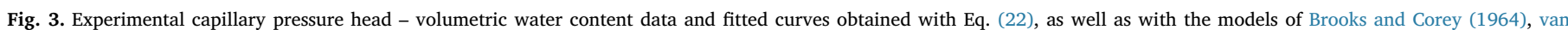

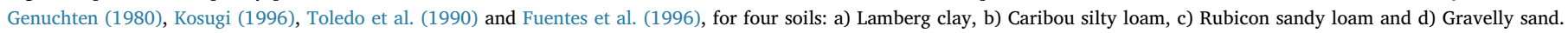


Table 1

Fitted parameters of the water retention functions of Alfaro Soto and Vilar (ASV), Brooks and Corey (BC), van Genuchten-Mualem, van Genuchten-Burdine and Kosugi, given by Eq. (20), Eq. (7) with $m=1-1 / n$, Eq. (7) with $m=1-2 / n$, and Eq. (10), respectively.

\begin{tabular}{|c|c|c|c|c|c|c|}
\hline \multirow{2}{*}{$\begin{array}{l}\text { Retention } \\
\text { Function }\end{array}$} & \multirow[t]{2}{*}{ Parameter } & \multirow[t]{2}{*}{ Unit } & \multicolumn{4}{|l|}{ Soil $^{2}$} \\
\hline & & & $\mathrm{a}$ & $\mathrm{b}$ & c & $\mathrm{d}$ \\
\hline \multirow[t]{5}{*}{$\mathrm{ASV}^{1}$} & $\theta_{s}$ & $(-)$ & 0.544 & 0.442 & 0.382 & 0.324 \\
\hline & $\theta_{r}$ & $(-)$ & 0.026 & 0.195 & 0.155 & 0.071 \\
\hline & $h_{o}$ & $(\mathrm{~cm})$ & 997 & 108 & 80 & 140 \\
\hline & $D$ & $(-)$ & 2.50 & 2.61 & 0.70 & 1.87 \\
\hline & $R^{2}$ & $(-)$ & 0.962 & 0.990 & 0.999 & 0.994 \\
\hline \multirow[t]{5}{*}{ BC } & $\theta_{s}$ & $(-)$ & 0.531 & 0.438 & 0.381 & 0.312 \\
\hline & $\theta_{r}$ & $(-)$ & 0.000 & 0.242 & 0.156 & 0.071 \\
\hline & $h_{a}$ & $(\mathrm{~cm})$ & 2.6 & 66.7 & 66.7 & 47.6 \\
\hline & $\lambda$ & $(-)$ & 0.460 & 0.569 & 2.310 & 1.126 \\
\hline & $R^{2}$ & $(-)$ & 0.973 & 0.996 & 0.999 & 0.989 \\
\hline \multirow[t]{6}{*}{ VGM } & $\theta_{s}$ & $(-)$ & 0.533 & 0.440 & 0.383 & 0.322 \\
\hline & $\theta_{r}$ & $(-)$ & 0.000 & 0.306 & 0.177 & 0.081 \\
\hline & $\alpha$ & $\left(\mathrm{cm}^{-1}\right)$ & 0.177 & 0.009 & 0.011 & 0.015 \\
\hline & $N$ & $(-)$ & 1.79 & 3.05 & 6.88 & 2.96 \\
\hline & $m=1-1 / n$ & $(-)$ & 0.442 & 0.672 & 0.855 & 0.662 \\
\hline & $R^{2}$ & $(-)$ & 0.998 & 0.999 & 0.990 & 0.999 \\
\hline \multirow[t]{6}{*}{ VGB } & $\theta_{s}$ & $(-)$ & 0.528 & 0.440 & 0.382 & 0.320 \\
\hline & $\theta_{r}$ & $(-)$ & 0.000 & 0.299 & 0.174 & 0.078 \\
\hline & $\alpha$ & $\left(\mathrm{cm}^{-1}\right)$ & 0.262 & 0.010 & 0.012 & 0.017 \\
\hline & $n$ & $(-)$ & 2.60 & 3.53 & 6.97 & 3.58 \\
\hline & $m=1-2 / n$ & $(-)$ & 0.231 & 0.434 & 0.713 & 0.442 \\
\hline & $R^{2}$ & $(-)$ & 0.994 & 1.000 & 0.992 & 0.998 \\
\hline \multirow[t]{5}{*}{ K } & $\theta_{s}$ & $(-)$ & 0.536 & 0.440 & 0.382 & 0.322 \\
\hline & $\theta_{r}$ & $(-)$ & 0.000 & 0.312 & 0.178 & 0.084 \\
\hline & $h_{o}$ & $(\mathrm{~cm})$ & 12.0 & 135.7 & 90.7 & 84.7 \\
\hline & $\sigma$ & $(-)$ & 1.207 & 0.587 & 0.264 & 0.645 \\
\hline & $R^{2}$ & $(-)$ & 0.999 & 0.999 & 0.990 & 0.998 \\
\hline
\end{tabular}

${ }^{1}$ ASV: Alfaro Soto and Vilar (2006); BC: Brooks and Corey (1964); VGM: van Genuchten (1980)-Mualem; VGB: van Genuchten (1980)-Burdine; K: Kosugi (1996).

${ }^{2}$ (a) Lamberg clay; (b) Caribou silty loam; (c) Rubicon sandy loam; (d) Gravelly sand.

are involved (Eqs. 13 and 23a). Because of the different retention equations, the $K_{r}(h)$ functions will be different also for the two models. The fact that the same $K_{r}\left(S_{e}\right)$ expressions are obtained is a consequence of the assumed scaling properties of the Burdine and Mualem theories (Eqs. (1) and (2), respectively), which are forcing the predictive conductivity curves to end at $K_{r}\left(S_{e}\right)=1$ when $S_{e}=1$. In other words, the multipliers of the $S_{e}(h)$ expressions (i.e., of Eqs. 13 and 23a) can be different, but not the exponents of $S_{e}$ in the two retention equations.

Figs. 4 and 5 shows plots of the relative hydraulic conductivity $\left(K_{r}\right)$ versus relative saturation $(S)$ calculated with Eq. (27), using the same

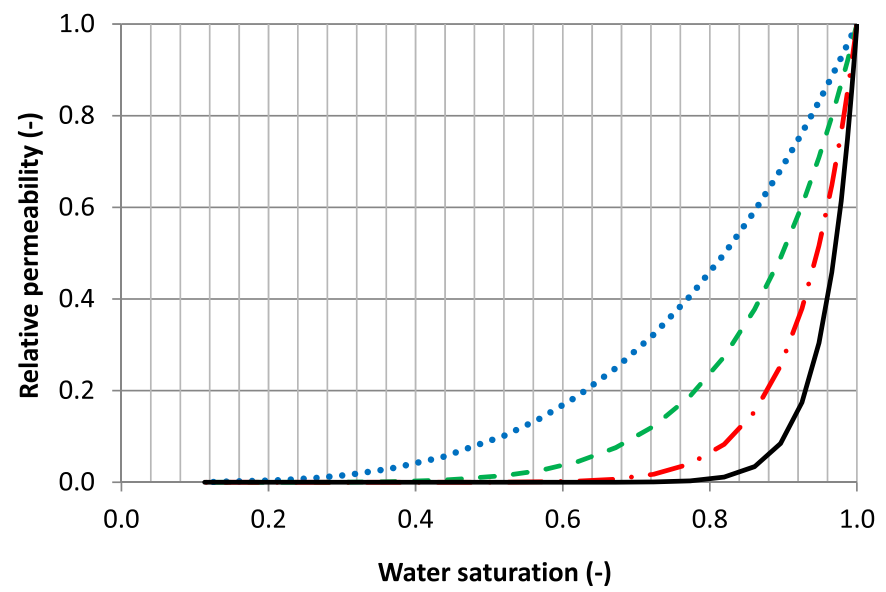

$\ldots \mathrm{D}=1.0 \quad-\mathrm{D}=2.5 \quad \mathrm{D}=2.8 \quad \mathrm{D}=2.9$

Fig. 4. Wetting phase relative permeability $\left(K_{r}\right)$ - relative saturation $(S)$ curves calculated with Eq. (27), based on Mualem's pore size distribution model assuming the same values for $S_{r}(0.23)$ and $h_{o}(0.5 \mathrm{~m})$ as in Fig. 1, and with $D$ varying between 1.0 and 2.9.

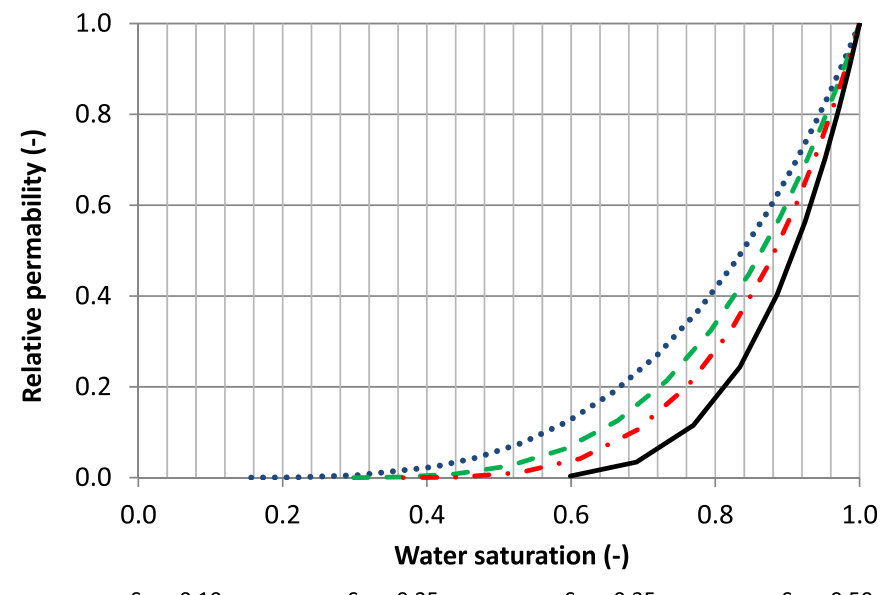

Fig. 5. Wetting phase relative permeability $\left(K_{r}\right)$ - relative saturation $(S)$ curves calculated with Eq. (27), based on Mualem's pore size distribution model assuming the same values for $h_{o}(0.5 \mathrm{~m})$ and $D(1.0)$ as in Fig. 2, with $S_{r}$ varying between 0.1 and 0.5.

parameters as in Figs. 1 and 2 respectively (i.e., $h_{o}=0.5 \mathrm{~m}, S_{r}=0.23$ and $D$ varying between 1.0 and 2.9 in Fig. 4 and $D=1$ and $S_{r}$ varying between 0.1 and 0.50 in Fig. 5). The plots in Fig. 4 show a considerable decrease in $K_{r}$ as the fractal dimension $(D)$ increases, leading to much steeper curves. For the theoretical upper limit of 3.0 for $D$, the curve would become a step function, being 1 at full saturation, and zero elsewhere. This situation is very similar to the traditional van Genuchten-Mualem and van Genuchten-Burdine equations, which have lower limits of the van Genuchten $n$ values of 1 and 2, respectively (van Genuchten and Nielsen, 1985; Vogel et al., 2001).

Fig. 5 shows that, as expected, an increase in residual saturation $\left(S_{r}\right)$ forces the wetting relative permeability curves to be restricted to increasingly smaller parts of relative saturation close to saturation.

\subsection{Example calculations of the relative hydraulic conductivity}

Eqs. (25) and (26) were used next to obtain the relative permeability of the wetting phase using the Burdine and Mualem approaches, respectively. The parameters $S_{r}, h_{o}$ and $D$ in the equations (Table 1) were estimated from the water retention curves shown in Fig. 3 using nonlinear least-squares. The accuracy of the fits were quantified by means of the root-mean-square deviation (RMSD) between the modeled and measured values. Values of RMSD close to zero provide better predictions, with larger values indicating greater deviations from measured values. Table 2 summarizes the results of the RMSD values obtained for each soil using the following equation:

Table 2

Root Mean Square Deviation (RMSD) values of predicted relative hydraulic conductivity functions using the pore size distribution models of Mualem and Burdine.

\begin{tabular}{|c|c|c|c|c|c|c|c|}
\hline \multirow[t]{2}{*}{ Soil } & \multirow{2}{*}{$\begin{array}{l}\text { Mualem } \\
\text { (1976b) } \\
\text { catalogue } \\
\text { number }\end{array}$} & \multirow[t]{2}{*}{ Soil name } & \multirow{2}{*}{$\begin{array}{l}\text { Conductivity } \\
\text { model }^{1}\end{array}$} & \multicolumn{4}{|c|}{ Model $^{2}$} \\
\hline & & & & ASV & $\mathrm{BC}$ & VG & K \\
\hline \multirow[t]{2}{*}{ a } & \multirow[t]{2}{*}{ 1003B } & Lamberg & M & 0.40 & 0.46 & 0.36 & 0.41 \\
\hline & & clay & B & 0.31 & 0.37 & 0.29 & 0.32 \\
\hline \multirow[t]{2}{*}{ b } & \multirow[t]{2}{*}{3301} & Caribou & M & 0.12 & 0.16 & 0.88 & 0.87 \\
\hline & & silty loam & B & 0.13 & 0.18 & 0.66 & 0.96 \\
\hline \multirow[t]{2}{*}{ c } & \multirow[t]{2}{*}{3501} & Rubicon & M & 0.25 & 0.26 & 1.26 & 1.27 \\
\hline & & sandy loam & B & 0.40 & 0.41 & 1.29 & 1.41 \\
\hline \multirow[t]{2}{*}{ d } & \multirow[t]{2}{*}{4135} & Gravelly & M & 0.09 & 0.12 & 0.07 & 0.08 \\
\hline & & sand G.E.9 & B & 0.10 & 0.09 & 0.13 & 0.15 \\
\hline
\end{tabular}

\footnotetext{
${ }^{1}$ M: Mualem (1976a), B: Burdine (1953).

${ }^{2}$ ASV: Alfaro Soto and Vilar (2006); BC: Brooks and Corey (1964); VG: van Genuchten (1980); K: Kosugi (1996).
} 


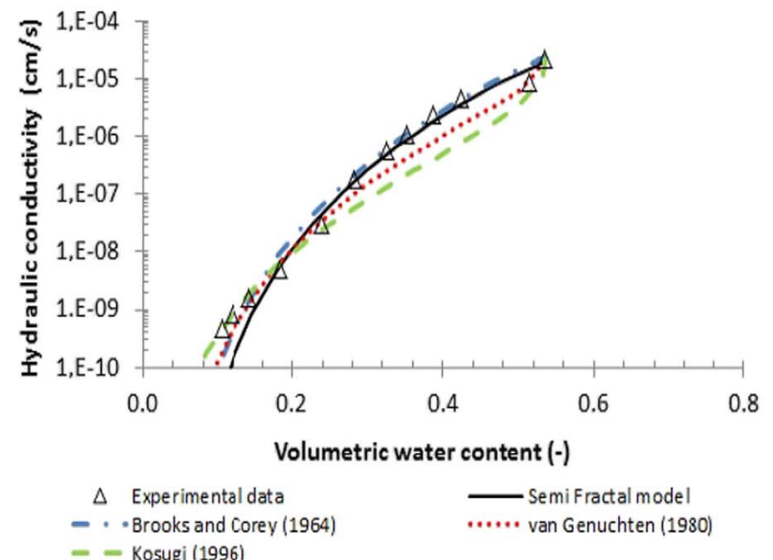

a

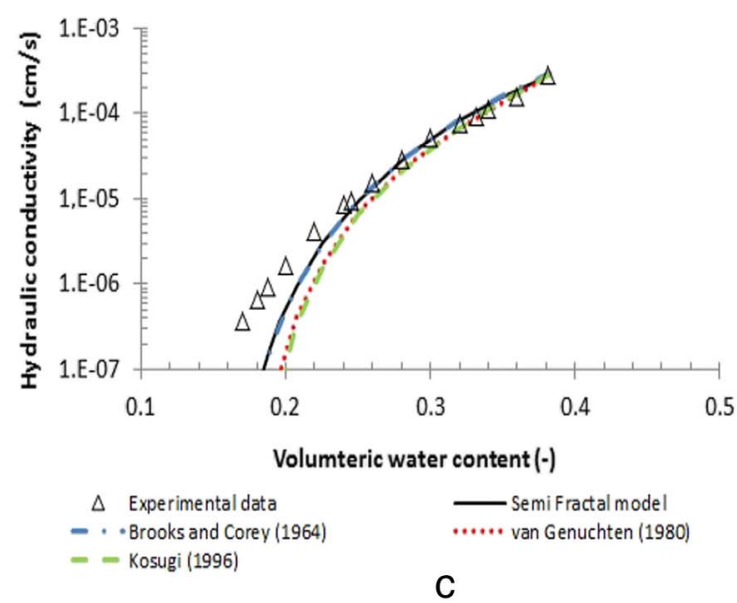

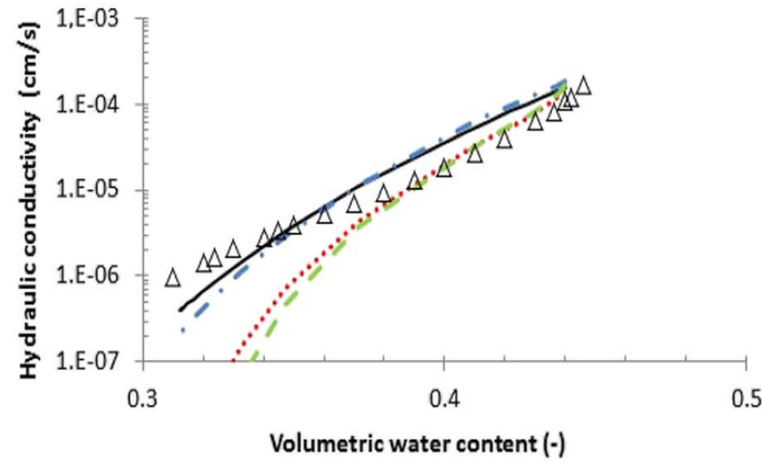

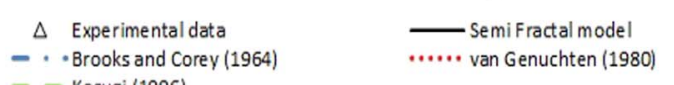

- Kosugi (1996)

b

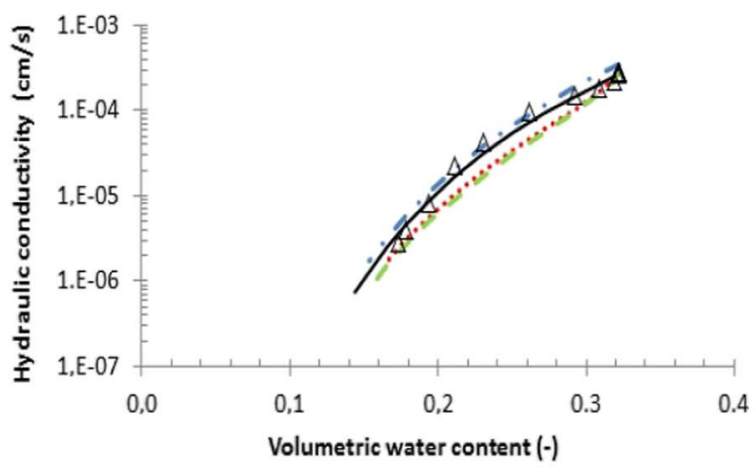

$\Delta \quad$ Experimental data
- - Brooks and Corey (1964)
- - Kosugi (1996) - Semi Fractal model

d

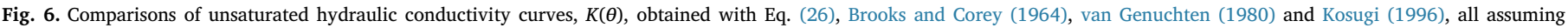
applicability of Burdine's (1953) model, and experimental data for four soils: a) Lamberg clay, b) Caribou silty loam, c) Rubicon sandy loam and d) Gravelly sand.

$\operatorname{RMSD}=\sqrt{\left[\log \left(K_{m}\right)-\log \left(K_{p}\right)\right]^{2} /(n-1)}$

where $K_{m}$ and $K_{p}$ represent, respectively, the measured and predicted relative permeability and $n$ represents the number of samples. The log $(K)$ values were used since the conductivity data follow a log-normal distribution (Kolmogorov-Smirnov statistical test using a significance level of $5 \%$ for all comparisons).

Fig. 6 shows predicted curves of the hydraulic conductivity, $K(\theta)$, for the same four soils as in Fig. 3. The plots compare the proposed Burdine-based permeability model (Eq. (25)) with the Burdine-based equations by Brooks and Corey (Eq. (5)), van Genuchten (Eq. (8)) and Kosugi (Eq. (11)), as well as with the experimental data as documented by Mualem (1976b) where the saturated hydraulic conductivity were: $2.25 \times 10^{-5} \mathrm{~cm} / \mathrm{s}, \quad 1.66 \times 10^{-4} \mathrm{~cm} / \mathrm{s}, \quad 2.83 \times 10^{-4} \mathrm{~cm} / \mathrm{s}$ and $2.78 \times 10^{-4} \mathrm{~cm} / \mathrm{s}$ in soils: a, b, c and d, respectively.

The results in Fig. 6 indicate close agreement between the $K$ predictions with the fractal-based model and the experimental data, as well as those obtained with the other Burdine based models. The fractal model agreed very well with the experimental data of especially soils $b$, $c$, and $d$. RMSD values for the fractal-based model were lowest in most cases as shown by the entries in Table 2. The RMSD values suggest that, overall for our soils, Eq. (24) performed best, followed by the models of Brooks and Corey, van Genuchten and Kosugi.

Similar results as in Fig. 6 are shown in Fig. 7 for the Mualem-based $K(\theta)$ predictions obtained using Eq. (26). The fractal model again performed better in most cases as reflected by the RMSD values in Table 2. The van Genuchten and Kosugi models for Rubicon sandy loam (soil c) in particular produced relatively poor results using either the Burdine or Mualem based expressions in Figs. 6 and 7, respectively.
In view of the results in Figs. 3, 6 and 7, we believe that the proposed fractal formulation for the water retention curve in combination with the Mualem or Burdine permeability models provide an possible alternative for describing the unsaturated soil hydraulic properties. Additional comparisons with measured data of different soil are clearly needed. The accuracy of the $K(\theta)$ predictions may well be a function of soil texture, with some studies suggesting that Burdine's model is equally or more accurate than Mualem's model for very coarse-textured soils, while the reverse may be true for medium and fine-textured systems (van Genuchten and Nielsen, 1985; Haverkamp and Parlange, 1986; API, 2007).

Although the models presented here (Eqs. (25) and (26)) provide reasonably accurate predictions of $\theta(h)$ and $K(\theta)$, we note that the predictions are based on a retention model that still assumes a constant saturation until the air-entry pressure. As mentioned earlier, this situation may not be realistic for certain field soils having relatively broad pore-size or particle-size distributions (van Genuchten and Nielsen, 1985; others). This restriction will also affect predictions of the hydraulic conductivity if the description of the retention curve near saturation is imperfect.

Also, our results indicate that Eqs. (25) and (26) tend to underestimate the measured conductivity data in the dry range, although this also occurred with the other models discussed here. Some of the discrepancy may have been due to difficulty in describing the water retention curve on the basis of a single fractal dimension $(D)$ applied to the entire curve. Although Eq. (21) is not linear, the model is based on a constant fractal dimension, which appears to be a limitation of many soils showing multifractal characteristics in their pore or particle size 

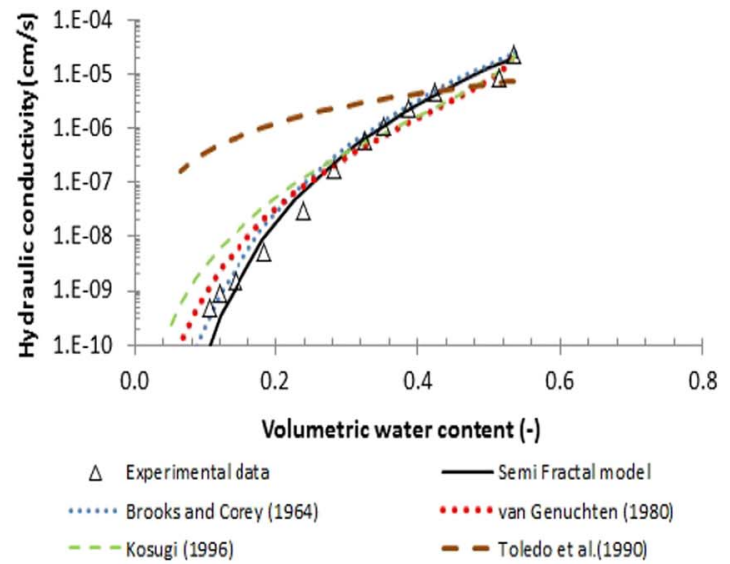

a

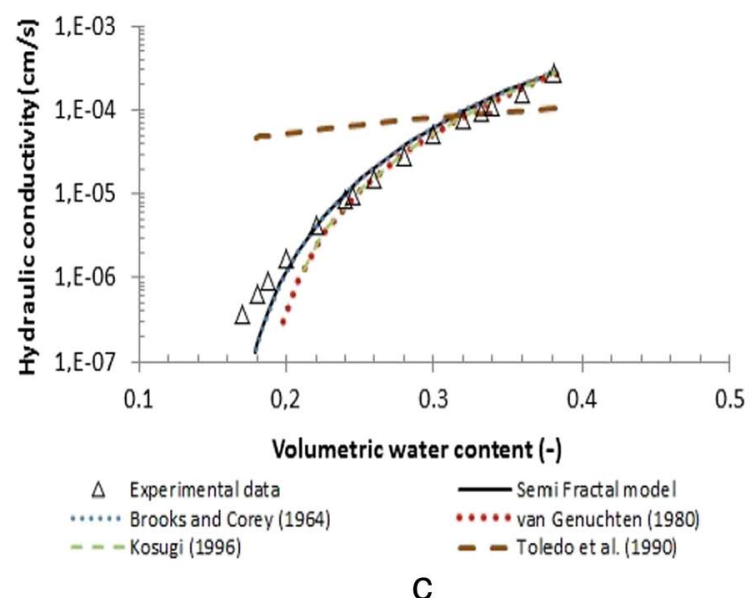

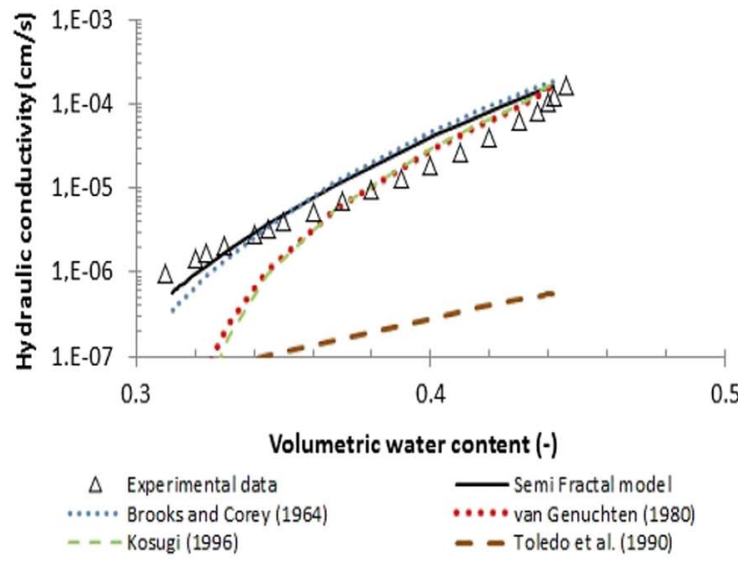

b

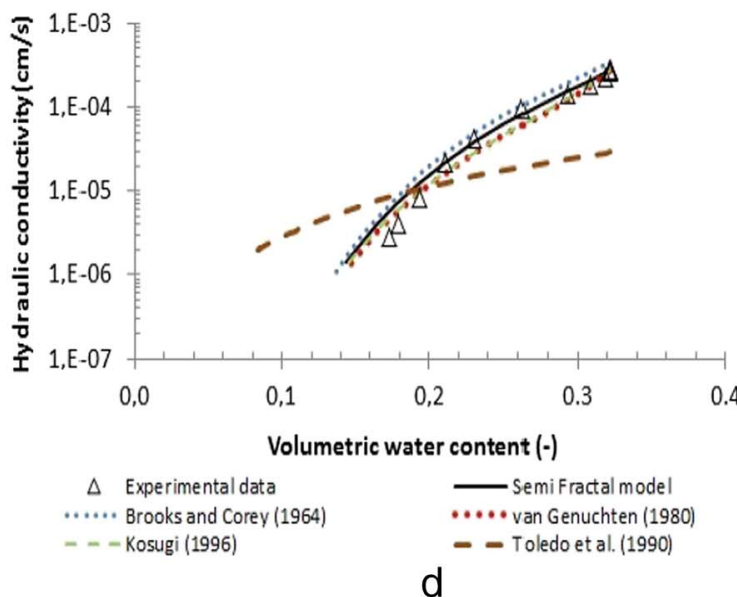

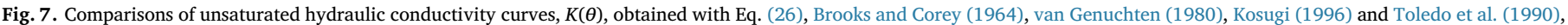
all assuming applicability of Mualem's (1976a) model, and experimental data for four soils: a) Lamberg clay, b) Caribou silty loam, c) Rubicon sandy loam and d) Gravelly sand.

distributions. Several studies (e.g., Grout et al., 1998; Posadas et al., 2001; Wang et al., 2008) have pointed out that multifractal concepts are generally very well suited for the particle size distribution of many soils, especially when fine-textured. Since pore size and particle size are closely correlated, the same may be true also for the water retention curve.

Multifractal models may be especially attractive for tropical soils, such as latosols and oxisols, which are more heterogeneous in their pore size distribution than many temperate soils. Measurement protocols and other factors may play a role also as explained well in a recent paper by Bieganowski et al. (2013) for the particle size distribution. As multifractal approaches have proliferated for the particle-size distribution, similar philosophies may be beneficial also for the soil hydraulic properties. Our approach in this paper still uses one fractal dimension, but implemented in a nonlinear manner over the entire retention function. In view of the shortcomings of having an air-entry value in the retention formulation, as done also in the classical Brooks and Corey model, perhaps a multi-fractal approach may well prove to be more promising.

Alternatively one could also implement more flexible but purely empirical retention functions, such as Eq. (7) as originally used by van Genuchten (1980) with independent $m$ and $n$ values. As shown by van Genuchten and Nielsen (1985), the presence of independent $m$ and $n$ values assumes that the curvatures of the curve near saturation at the air entry value will be independent of the curvature in the dry side of the curve. van Genuchten and Nielsen (1985) showed that this form of the retention function is always more accurate than the Books and Corey and restricted van Genuchten-Mualem and van Genuchten-Burdine functions. While we did not further investigate this problem here using our fractal-based functions, Eq. (7) with independent $m$ and $n$ values may well be more accurate than most or all approaches, including our formulation. While the resulting hydraulic conductivity models in combination with Eq. (7) are definitely more complicated, the recent introduction of advanced mathematical packages such as Mathematica, Matlab or Mathcad with their tool boxes of special functions (in particular, the incomplete beta and hypergeometric functions) should soften this limitation. An even more far-reaching approach would be to implement form-free descriptions of the hydraulic properties as shown by Iden and Durner (2007).

\section{Conclusions}

In this paper we presented and tested two new models based on fractal geometry designed to predict the relative permeabilities of the wetting phases in two-phase systems for different types of soils. The models assume a more realistic fractal based description of the water retention curve, in conjunction with the statistical pore-size distribution models of Burdine (1953) and Mualem (1976a). The proposed models are different from those based on purely empirical formulations of the water retention curve that do not necessarily reflect our understanding of the interaction between water and soil. The proposed formulations showed close agreement with existing models for the wetting permeabilities, as well as with measured experimental data for four different soil types. An advantage of the models is their applicability over the full range of pore sizes in a soil. They appear useful tool for studying and describing the hydraulic properties of soils and rocks. 


\section{Acknowledgements}

This work was supported by the Fundação para o Desenvolvimento da Unesp - FUNDUNESP, São Paulo, Brazil.

\section{References}

Adler, P.M., 1992. Porous Media: Geometry and Transports. Butterworth and Heinemann, Stoneham, MA.

Alfaro Soto, M.A., Vilar, O.M., 2006. Evaluation of a pore fractal model for the prediction of soil water retention curve. In: Miller, G.A., Zapata, C.A., Houston, S.L., Fredlund, D.G. (Eds.), Unsaturated Soils 2006. ASCE Geotechnical Special Publ. 147 2. pp. 2441-2452. http://dx.doi.org/10.1061/40802(189)208.

API, 2007. Light Non-aqueous Phase Liquid Distribution and Recovery Model (LDRM). Publication 4760. V.1,2. American Petroleum Institute, Washington, DC.

Assouline, S., Tartakovsky, D.M., 2001. Unsaturated hydraulic conductivity function based on a fragmentation process. Water Resour. Res. 37 (5), 1309-1312.

Bieganowski, A., Chojeckib, T., Sochan, A., Lamorski, K., 2013. Methodological aspects of fractal dimension estimation on the basis of particle size distribution. Vadose Zone J. 12 (1). http://dx.doi.org/10.2136/vzj2012.0064.

Bird, N.R.A., 1998. Comment on "An improved fractal equation for the soil water retention curve" by E. Perfect et al. Water Resour. Res. 34, 929-930.

Brooks, R.H., Corey, A.T., 1964. Hydraulic properties of porous media. In: Hydrology Paper 3. Colorado State Univ, Fort Collins, CO.

Burdine, N.T., 1953. Relative permeability calculation size distribution data. Trans. Am. Inst. Min. Metall. Eng. 198, 71-78.

Campbell, G.S., 1974. A simple method for determining unsaturated conductivity from soil moisture retention data. Soil Sci. 117, 311-314.

Carsel, R.F., Parrish, R.S., 1988. Developing joint probability distributions of soil water retention characteristics. Water Resour. Res. 24 (5), 755-769.

Childs, E.C., Collis-George, N., 1950. The permeability of porous materials. Proc. R. Soc., Ser. A 201, 392-405.

Cihan, A., Perfect, E., Tyner, J.S., 2009. Predicting relative permeability from water retention: a direct approach based on fractal geometry. Water Resour. Res. 45, W04404. http://dx.doi.org/10.1029/2008WR007038.

Dane, J.H., Topp, G.C., 2002. Methods of soil analysis, part 4, physical methods. In: SSSA Book Series 5. Soil Science Soc. Am., Madison, WI (2002).

Dourado Neto, D., de Jong van Lier, Q., van Genuchten, M.Th., Reichardt, K., Metselaar, K., Nielsen, D.R., 2011. Alternative analytical expressions for the general van Genuchten-Mualem and van Genuchten-Burdine hydraulic conductivity models. Vadose Zone J. 10, 618-623. http://dx.doi.org/10.2136/vzj2009.0191.

Durner, W., 1994. Hydraulic conductivity estimation for soils with heterogeneous pore structure. Water Resour. Res. 32 (9), 211-223.

Fredlund, D.G., Xing, A., Huang, S., 1994. Predicting the permeability function for unsaturated soils using the soil-water characteristic curve. Can. Geotech. J. 31, $533-546$.

Fuentes, C., Vauclin, M., Parlange, J.-Y., Haverkamp, R., 1996. A note on the soil-water conductivity of a fractal soil. Transp. Porous Media 23, 31-36.

van Genuchten, M.Th., 1980. A closed-form equation for predicting the hydraulic conductivity of unsaturated soils. Soil Sci. Soc. Am. J. 44, 892-898.

van Genuchten, M.Th., Nielsen, D.R., 1985. On describing and predicting the hydraulic properties of unsaturated soils. Ann. Geophys. 3 (5), 615-628.

Ghanbarian-Alavijeh, B., Hunt, A.H., 2012a. Comments on "More general capillary pressure and relative permeability models from fractal geometry" by Kewen Li. J. Contam. Hydrol. 140-141, 21-23.
Ghanbarian-Alavijeh, B., Hunt, A.H., 2012b. Unsaturated hydraulic conductivity in porous media: percolation theory. Geoderma 187-188, 77-84.

Ghanbarian-Alavijeh, B., Liaghat, A., Huang, G.-H., van Genuchten, M.Th., 2010. Estimation of the van Genuchten soil water retention properties from soil textural data. Pedosphere 20 (4), 456-465.

Giménez, D., Perfect, E., Rawls, W.J., Pachepsky, Y., 1997. Fractal models for predicting soil hydraulic properties: a review. Eng. Geol. 48 (1-3), 161-183.

Grout, H., Tarquis, A.M., Wisner, M.R., 1998. Multifractal analysis of particle size distributions in soil. Environ. Sci. Technol. 32, 1176-1182.

Haverkamp, R., Parlange, J.-Y., 1986. Predicting the water retention curve from particle size distribution: I. Sandy soils without organic matter. Soil Sci. 142, 325-339.

Hunt, A.G., 2004. Comparing van Genuchten and percolation theoretical formulations of the hydraulic properties of unsaturated media. Vadose Zone J. 3, 1483-1488.

Iden, S.C., Durner, W., 2007. Free-form estimation of the unsaturated soil hydraulic properties by inverse modeling using global optimization. Water Resour. Res. 43, W07451. http://dx.doi.org/10.1029/2006WR005845.

Kosugi, K., 1996. Lognormal distribution model for unsaturated soil hydraulic properties. Water Resour. Res. 32 (9), 2697-2703.

Leij, F.J., Russell, W.B., Lesch, S.M., 1997. Closed-form expressions for water retention and conductivity data. J. Hydrol. 35 (5), 848-858.

Li, K., 2010. More general capillary pressure and relative permeability models from fractal geometry. J. Contam. Hydrol. 111 (1-4), 13-24.

Millington, R.J., Quirk, J.P., 1961. Permeability of porous solids. Trans. Faraday Soc. 57, 1200-1206.

Mualem, Y., 1976a. A new model for predicting the hydraulic conductivity of unsaturated porous media. Water Resour. Res. 12, 593-622.

Mualem, Y., 1976b. A catalogue of the hydraulic properties of unsaturated soils. In: Research Project Report No 442. Haifa, Technion, Israel Institute of Technology.

Perfect, E., 1999. Estimating soil mass fractal dimensions from water retention curves. Geoderma 88, 221-231.

Posadas, A.D., Gimenez, P.D., Bittelli, M., Vaz, C.M.P., Flury, M., 2001. Multifractal characterization of soil particle-size distributions. Soil Sci. Soc. Am. J. 65, 1361-1367.

Purcell, W.R., 1949. Capillary pressures, their measurement using mercury and the calculation of permeability therefrom. J. Pet. Technol. 1, 39-46.

Rieu, M., Sposito, G., 1991. Fractal fragmentation, soil porosity and soil water properties. I. Theory. Soil Sci. Soc. Am. J. 55, 1231-1244.

Schaap, M.G., Leij, F.J., van Genuchten, M.Th., 2001. Rosetta: a computer program for estimating soil hydraulic parameters with hierarchical pedotransfer functions. J. Hydrol. 251, 163-176.

Toledo, P.G., Novy, R.A., Davis, H.T., Scriven, L.E., 1990. Hydraulic conductivity of porous media at low water content. Soil Sci. Soc. Am. J. 54, 673-679.

Tyler, S.W., Wheatcraft, S.W., 1990. Fractal processes in soil water retention. Water Resour. Res. 26 (5), 1047-1054.

Vereecken, H., Weynants, M., Javaux, M., Pachepsky, Y., Schaap, M.G., van Genuchten, M.Th., 2010. Using pedotransfer functions to estimate the van Genuchten-Mualem soil hydraulic properties: a review. Vadose Zone J. 9, 795-820.

Vogel, T., van Genuchten, M.Th., Cislerova, M., 2001. Effect of the shape of the soil hydraulic functions near saturation on variably-saturated flow predictions. Adv. Water Resour. 24 (2), 133-144.

Wang, D., Fu, B., Zhao, W., Hu, H., Wang, Y., 2008. Multifractal characteristics of soil particle size distribution under different land-use types on the Loess Plateau, China. Catena 72, 29-36.

Xu, Y., 2004. Calculation of unsaturated hydraulic conductivity using a fractal model for the pore-size distribution. Comput. Geotech. 31, 549-557.

Xu, Y., Dong, P., 2004. Fractal approach to hydraulic properties in unsaturated porous media. Chaos, Solitons Fractals 19, 327-337. 